\title{
REGIME SHIFT IN A PHYTOPLANKTON-PHOSPHORUS MODEL WITH VERTICAL STRUCTURE AND SEASONALITY
}

\author{
KOTA IKEDA AND TAKESHI MIKI
}

\begin{abstract}
Many ecological systems are influenced by positive feedbacks between organisms and abiotic environments, which generates multiple stable equilibria of a mathematical model with a hysteresis structure. In addition, discontinuous shifts of system at equilibrium is predicted, which is often called regime shift in ecosystem sciences. This hysteresis structure is unfavorable from environmental management point of view, because the reconstruction of original equilibrium state requests much lower levels of external forcing. Mathematical models proposed in previous works are simple and mathematically tractable ([7], [2]). However, it is difficult to extrapolate from such simple models the occurrence likelihood of regime shift in natural environments since temporally dynamic features in ecology and physico-chemical environments, and spatial dimension are less explored in those models.

In this study, we construct a realistic but mathematically tractable model of interaction between phytoplankton and phosphorus, which incorporates (1) 1-dimensional vertical structure of lake ecosystem and (2) seasonal periodic cycle of water mixing. We aim to understand the impact of changes in seasonality in various types of lakes on the occurrence of multiple attractors (periodic solution) and hysteresis structure.
\end{abstract}

\section{Introduction}

Many ecological systems such as grassland vegetation, lake food chain, and coral reef are influenced by positive feedbacks between organisms and abiotic environments. From dynamical point of view, multiple (locally) asymptotically stable equilibria (or alternative stable states) emerge when positive feedbacks are dominant processes in such systems ([6], [4], [3]). In addition, with multiple stable equilibria, along with continuous changes in external forcing (e.g. water availability in soil for grassland system or nutrient loading to lake ecosystem), discontinuous shifts of system at equilibrium is predicted (it is often called regime shift in ecosystem sciences). Furthermore, the critical level of the external forcing that induces the shift from state " 1 " to state " 2 " is different from the level that induces the return from state

Received July 08, 2015, accepted October 14, 2015.

2010 Mathematics Subject Classification. 92D40 (34K13, 34D20, 37G15).

Key words and phrases. Ecology, periodic solutions, stability, bifurcations.

Corresponding author: Kota Ikeda. 
"2" to state " 1 "; this is called hysteresis. This hysteresis structure is unfavorable from environmental management point of view, because the reconstruction of original equilibrium state requests much lower levels of external forcing.

Simple mathematical models have been proposed and mathematically analyzed ([7], [2]). One of the major target ecosystems of these models is a lake ecosystem; phosphorus is a growth limiting factor for phytoplankton. The increase of phosphorus loading from land to lake (i.e., external P loading) gradually enhances the phytoplankton production and biomass. The organic matter produced by phytoplankton is finally decomposed by microbes into carbon dioxide $\left(\mathrm{CO}_{2}\right)$ and inorganic phosphorus, which in turn consumed again by the phytoplankton for primary production. However, at the same time, microbial decomposition of organic matter consumes dissolved oxygen in water and sediment, which induces the release of iron- and aluminum-bounded phosphorus from the lake sediment into lake water (i.e., internal P loading, [8]). This acts as a positive feedback between internal P loading and phytoplankton growth, leading to the multiple stable states that can be realized with the identical external P loading and thus discontinuous shifts of equilibrium phytoplankton biomass and the hysteresis structure emerges along the continuous shifts in external P loading.

These mathematical models are very simple and mathematically tractable ([7], [2]). However, it is difficult to extrapolate from such simple models the occurrence likelihood of regime shift in natural environments, since models are generally neglect many complex features in nature (but see [5]). Implication of high risk of regime shifts (e.g. [1]) by simple mathematical models needs caution. There are two major problems in case for lake models. First, temporally dynamic features in ecology and physico-chemical environments are not considered in mathematical modeling. Many systems would have more diverse attractors e.g. multiple periodic solutions (seasonal patterns of phytoplankton bloom). Second, spatial dimension has less-explored (but see [5]); primary production at surface later (epilimnion), decomposition of organic matter and deletion of oxygen in deeper layers (hypolimnion) generally occur at the different depth of lake but usually non-spatial differential equations are used to describe phosphorus-phytoplankton dynamics. These two aspects are important not only from theoretical point view but also from rising risk of lake ecosystems under warming. Deep lake is highly dynamic in terms of water circulation and seasonality in plankton food chain. Lake water warming is changing the strength, depth, and seasonality of vertical water mixing. It is argued weaker and shorter vertical mixing in winter season under warming results in severer oxygen depletion in hypolimnion and sediment of lakes, which is more likely to induce internal $\mathrm{P}$ loading and regime shift.

In this study, we construct a realistic but mathematically tractable model of interaction between phytoplankton and phosphorus, which incorporates (1) 1-dimensional vertical 
structure of lake ecosystem and (2) seasonal periodic cycle of water mixing. We aim to understand the impact of changes in seasonality in various types of lakes on the occurrence of multiple attractors (periodic solution) and hysteresis structure.

\section{Model}

\section{Seasonally changing model}

We construct a model to describe phosphorus-phytoplankton dynamics. To study the seasonal change of phosphorus and phytoplankton concentrations, we assume that 1 year consists of 365 days which are divided into only summer and winter. Here we denote the period of summer by $T_{\mathrm{s}}$. Then the period of winter is given by $T_{\mathrm{w}}=365-T_{\mathrm{s}}$.

In order to simplify our model, we assume the horizontal homogeneity, which means that two state variables depend only on time and depth. Since vertical water mixing does not occur in summer, phosphorus and phytoplankton concentrations have spatial gradients. A lake consists of a epilimnion, a hypolimnion, and a bottom sediment, which depths are represented by $Z_{\mathrm{e}}, Z_{\mathrm{h}}$, and $Z_{\mathrm{b}}$, respectively. We study the dynamics of two state variables in each water column. The total depth of the lake is given by $Z=Z_{\mathrm{e}}+Z_{\mathrm{h}}+Z_{\mathrm{b}}$. The epilimnion is strongly influenced by wind and well-mixed. Thickness of the bottom sediment is narrower than the epilimnion and hypolimnion. Hence phosphorus and phytoplankton concentrations in the epilimnion and bottom sediment are assumed to be spatially constant. Since a whole lake is well-mixed by convection in winter, the two state variables do not have spatial gradients.

We denote the $k$-th year phosphorus and phytoplankton concentrations in the epilimnion and the hypolimnion, the bottom sediment in summer by $\left(P_{k}^{\mathrm{e}}, X_{k}^{\mathrm{e}}\right),\left(P_{k}^{\mathrm{h}}, X_{k}^{\mathrm{h}}\right)$, and $\left(P_{k}^{\mathrm{b}}, X_{k}^{\mathrm{b}}\right)$ $(k=1,2, \ldots)$. These variables are governed by the following system;

$$
\begin{aligned}
& \frac{\mathrm{d} P_{k}^{\mathrm{e}}}{\mathrm{d} t}=\frac{l}{Z_{\mathrm{e}}}-\frac{\alpha}{Z_{\mathrm{e}}^{\gamma-1}} P_{k}^{\mathrm{e}}+c_{1} \beta_{\mathrm{e}} X_{k}^{\mathrm{e}}, \quad 0<t<T_{\mathrm{s}}, \\
& \frac{\mathrm{d} X_{k}^{\mathrm{e}}}{\mathrm{d} t}=c_{2} \frac{\alpha}{Z_{\mathrm{e}}^{\gamma-1}} P_{k}^{\mathrm{e}}-\left(\beta_{\mathrm{e}}+\frac{s_{\mathrm{e}}}{Z_{\mathrm{e}}}\right) X_{k}^{\mathrm{e}}, \quad 0<t<T_{\mathrm{s}}, \\
& \frac{\partial P_{k}^{\mathrm{h}}}{\partial t}=c_{1} \beta_{\mathrm{h}} X_{k}^{\mathrm{h}}, \quad 0<t<T_{\mathrm{s}}, \quad 0<z<Z_{\mathrm{h}} \text {, } \\
& \frac{\partial X_{k}^{\mathrm{h}}}{\partial t}=-s_{\mathrm{h}} \frac{\partial X_{k}^{\mathrm{h}}}{\partial z}-\beta_{\mathrm{h}} X_{k}^{\mathrm{h}}, \quad 0<t<T_{\mathrm{s}}, \quad 0<z<Z_{\mathrm{h}} \text {, } \\
& \frac{\mathrm{d} P_{k}^{\mathrm{b}}}{\mathrm{d} t}=c_{1} \beta_{\mathrm{b}} X_{k}^{\mathrm{b}}+\frac{1}{Z_{\mathrm{b}}} f\left(u_{k}\right), \quad 0<t<T_{\mathrm{s}}, \\
& \frac{\mathrm{d} X_{k}^{\mathrm{b}}}{\mathrm{d} t}=\frac{s_{\mathrm{h}}}{Z_{\mathrm{b}}} X_{k}^{\mathrm{h}}\left(Z_{\mathrm{h}}, t\right)-\left(\beta_{\mathrm{b}}+\frac{s_{\mathrm{b}}}{Z_{\mathrm{b}}}\right) X_{k}^{\mathrm{b}}, \quad 0<t<T_{\mathrm{s}},
\end{aligned}
$$


Table 1: Parameter sets

\begin{tabular}{|c|c|c|c|c|}
\hline Symbols & Meaning & Units & Set I & Set II \\
\hline \multicolumn{5}{|l|}{ State variables } \\
\hline$X^{\mathrm{e}}, X^{\mathrm{h}}, X^{\mathrm{b}}, X$ & Chlorophyll concentration & $\mu \mathrm{g} \mathrm{P} \mathrm{L}^{-1}$ & & \\
\hline$P^{\mathrm{e}}, P^{\mathrm{h}}, P^{\mathrm{b}}, P$ & Phosphorus concentration & $\mu \mathrm{g} \mathrm{P} \mathrm{L}^{-1}$ & & \\
\hline \multicolumn{5}{|l|}{ Parameters } \\
\hline$l$ & P loading rate & $\mathrm{mg} \mathrm{P} \mathrm{m}^{-2} \mathrm{~d}^{-1}$ & $0.0-1.0$ & $0.0-10.0$ \\
\hline$\alpha$ & Phytoplankton growth rate & $\mathrm{d}^{-1}$ & 1.0 & 1.0 \\
\hline$\gamma$ & Light attenuation intensity & & 1.0 & 2.0 \\
\hline$c_{1}$ & P excretion rate associated with grazing & & 1.0 & 1.0 \\
\hline$c_{2}$ & Converting rate associated with $\mathrm{P}$ consuming & & 1.0 & 1.0 \\
\hline$\beta_{\mathrm{e}}, \beta_{\mathrm{h}}, \beta_{\mathrm{b}}, \beta$ & Zooplankton grazing rate & $\mathrm{d}^{-1}$ & 0.05 & 0.1 \\
\hline$s_{\mathrm{e}}, s_{\mathrm{h}}$ & Phytoplankton sinking rate & $\mathrm{m} \mathrm{d}^{-1}$ & 5.0 & 1.0 \\
\hline$s_{\mathrm{b}}$ & Phytoplankton sinking rate & $\mathrm{m} \mathrm{d}^{-1}$ & 2.5 & 2.5 \\
\hline$\kappa$ & Decaying rate of oxygen & $(\mu \mathrm{g} \mathrm{P})^{-1} \mathrm{~L} \mathrm{~d}^{-1}$ & 0.05 & 0.07 \\
\hline$u_{0}$ & Saturated oxygen concentration & $\mathrm{mg} \mathrm{L}^{-1}$ & 10.0 & 10.0 \\
\hline$\theta$ & $\begin{array}{l}\text { Threshold between oxic } \\
\text { and anoxic conditions }\end{array}$ & $\mathrm{mg} \mathrm{L}^{-1}$ & 3.0 & 3.0 \\
\hline$Z_{\mathrm{e}}$ & Depth of epilimnion & $\mathrm{m}$ & 10 & 10 \\
\hline$Z_{\mathrm{h}}$ & Depth of hypolimnion & $\mathrm{m}$ & 70 & 48 \\
\hline$Z_{\mathrm{b}}$ & Depth of bottom & $\mathrm{m}$ & 0.1 & 0.1 \\
\hline$Z=Z_{\mathrm{e}}+Z_{\mathrm{h}}+Z_{\mathrm{b}}$ & Total depth of lake & $\mathrm{m}$ & 80.1 & 58.1 \\
\hline$R$ & P recycling rate & $\operatorname{mg~P~m}{ }^{-2} \mathrm{~d}^{-1}$ & 10.5 & 9.6 \\
\hline$T_{\mathrm{S}}$ & Period of summer & $\mathrm{d}$ & & \\
\hline$T_{\mathrm{W}}=365-T_{\mathrm{s}}$ & Period of winter & $\mathrm{d}$ & & \\
\hline
\end{tabular}

where $f(u)$ is a step function defined by

$$
f(u)= \begin{cases}R, & u<\theta, \\ 0, & u>\theta .\end{cases}
$$

Oxygen concentration denoted by $u_{k}$ is governed by

$$
\left\{\begin{array}{l}
\frac{\mathrm{d} u_{k}}{\mathrm{~d} t}=-\kappa u_{k} X_{k}^{\mathrm{b}}, \quad 0<t<T_{\mathrm{s}} \\
u_{k}(0)=u_{0}
\end{array}\right.
$$

Also we denote phosphorus and phytoplankton concentrations in winter by $\left(P_{k}, X_{k}\right)$, which is governed by

$$
\begin{array}{ll}
\frac{\mathrm{d} P_{k}}{\mathrm{~d} t}=\frac{l}{Z}-\frac{\alpha}{Z^{\gamma-1}} P_{k}+c_{1} \beta X_{k}, & 0<t<T_{\mathrm{w}}, \\
\frac{\mathrm{d} X_{k}}{\mathrm{~d} t}=c_{2} \frac{\alpha}{Z^{\gamma-1}} P_{k}-\left(\beta+\frac{s_{\mathrm{b}}}{Z}\right) X_{k}, & 0<t<T_{\mathrm{w}} .
\end{array}
$$

Phytoplankton sinks down from the top to the bottom with sinking rates $s_{\mathrm{e}}, s_{\mathrm{h}}, s_{\mathrm{b}}\left[\mathrm{m} \mathrm{d}^{-1}\right]$ and disappears due to grazing by zooplankton with grazing rates $\beta_{\mathrm{e}}, \beta_{\mathrm{h}}, \beta_{\mathrm{b}}, \beta\left[\mathrm{d}^{-1}\right]$. We as- 
sume that these two mechanisms of sinking and grazing affect the dynamics of two state variables commonly in all water columns throughout years. Since environmental conditions in each column and season may be different, the parameters are given individually. $\mathrm{P}$ excretion rate associated with grazing is represented by $c_{1}$. Inputs of phosphorus include P loading from the watershed in epilimnion and $\mathrm{P}$ recycling from the bottom sediment. As stated in [5], we think of this $\mathrm{P}$ loading as a controllable variable denoted by $l\left[\mathrm{mg} \mathrm{P} \mathrm{m}^{-2} \mathrm{~d}^{-1}\right]$ in terms of restoration management. Hence the parameter $l$ is a main parameter in this article. If phosphorus is not supplied from the surface of the lake, phosphorus and phytoplankton concentrations must decrease with time. Hence we assume a sufficient condition such as $0 \leq c_{1} \leq 1$ and $0 \leq c_{2} \leq 1$. The growth rate of phytoplankton is given by $c_{2} \alpha / Z_{\mathrm{e}}^{\gamma-1}$ and depends on the depth of epilimnion $Z_{\mathrm{e}}$ with converting rate $c_{2}$ and light attenuation intensity $\gamma$, which represents a degree of suspension in the lake.

Phosphorus recycling from the sediment is assumed to occur when dissolved oxygen in the bottom sediment decreases sufficiently. A threshold between oxic and anoxic conditions is given by $\theta\left[\mathrm{mg} \mathrm{L}^{-1}\right]$. An input of phosphorus recycling is assumed to be constant $R$ [mg $\mathrm{P} \mathrm{m}^{-2} \mathrm{~d}^{-1}$ ] under the anoxic condition. This nonlinear effect is represented by a function $f(u)$. In [5], $R$ is defined as a nonlinear function depending on phytoplankton density $X$ and temperature. The explicit form of $R$ in [5] gives us an estimate of $0 \leq R \leq 14$. In particular, we have $R=9.88$ for $X=40$ and $20^{\circ} \mathrm{C}$.

The dynamics of oxygen concentration in summer is assumed to be governed by the mass action law and decay exponentially with a rate $\kappa X_{k}^{\mathrm{b}}$. On the other hand, we do not consider either the dynamics of oxygen concentration or phosphorus recycling from the sediment in winter because water is well-mixed vertically and oxygen is sufficiently supplied from the surface of the lake. This also implies that the initial condition of oxygen concentration in summer is given by $u_{0}>\theta$ independent of $k$.

The initial values of phosphorus and phytoplankton concentrations for the $k$-th year are given by

$$
\begin{aligned}
& P_{k}^{\mathrm{e}}(0)=P_{k}^{\mathrm{h}}(z, 0)=P_{k}^{\mathrm{b}}(0)=P_{k-1}\left(T_{\mathrm{w}}\right), \quad X_{k}^{\mathrm{e}}(0)=X_{k}^{\mathrm{h}}(z, 0)=X_{k}^{\mathrm{b}}(0)=X_{k-1}\left(T_{\mathrm{w}}\right), \\
& P_{k}(0)=\frac{1}{Z}\left(Z_{\mathrm{e}} P_{k}^{\mathrm{e}}\left(T_{\mathrm{s}}\right)+\int_{0}^{Z_{\mathrm{h}}} P_{k}^{\mathrm{h}}\left(z, T_{\mathrm{s}}\right) \mathrm{d} z+Z_{\mathrm{b}} P_{k}^{\mathrm{b}}\left(T_{\mathrm{s}}\right)\right), \\
& X_{k}(0)=\frac{1}{Z}\left(Z_{\mathrm{e}} X_{k}^{\mathrm{e}}\left(T_{\mathrm{s}}\right)+\int_{0}^{Z_{\mathrm{h}}} X_{k}^{\mathrm{h}}\left(z, T_{\mathrm{s}}\right) \mathrm{d} z+Z_{\mathrm{b}} X_{k}^{\mathrm{b}}\left(T_{\mathrm{s}}\right)\right),
\end{aligned}
$$

where we denote $P_{0}\left(T_{\mathrm{w}}\right)=P_{0}$ and $X_{0}\left(T_{\mathrm{w}}\right)=X_{0}$ for $P_{0}$ and $X_{0}$ given initially.

The dynamics of phosphorus and phytoplankton concentrations begins with (2.1)-(2.4) and (2.6) for $k=1$. When the time achieves $t=T_{\mathrm{s}}$, we calculate $\left(P_{1}(0), X_{1}(0)\right)$ by (2.7). Next we consider (2.5) for $k=1$ and have $\left(P_{1}\left(T_{\mathrm{w}}\right), X_{1}\left(T_{\mathrm{W}}\right)\right)$, which generates the initial values of 


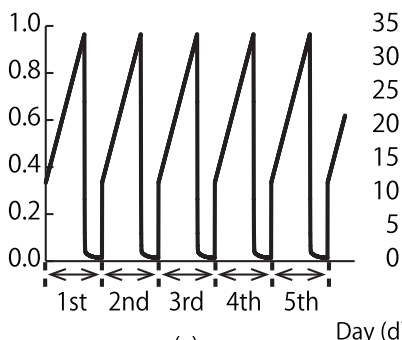

(a)

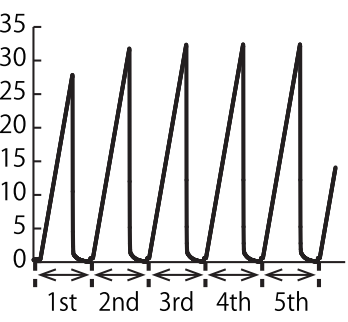

(b)

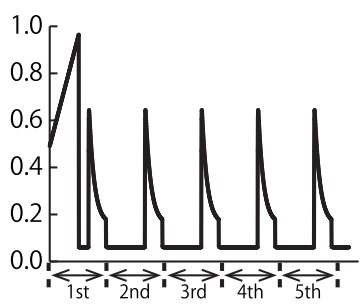

(c)

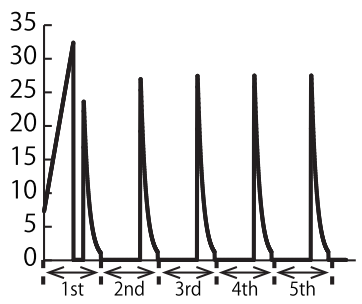

(d)

Figure 1: Bistability in the system (2.1)-(2.7). Figures (a) and (c) correspond to time series of a solution in the system (2.1) $-(2.7)$ for $T_{\mathrm{s}}=251$ and $l=0.4$ with a random initial value $\left(P_{0}, X_{0}\right)$. Figures (b) and (d) also correspond to time series of a solution under almost the same condition as in Figures (a) and (c). The difference between two conditions is only on the initial values. We used the parameters of Set I in Table 1 in both cases. In the figures we plot $\left(\bar{P}_{k}(t-365(k-1)), \bar{X}_{k}(t-365(k-1))\right)$ in 365(k-1)<t< $T_{\mathrm{S}}+365(k-1)$ and $\left(P_{k}\left(t-T_{\mathrm{s}}-\right.\right.$ $\left.365(k-1)), X_{k}\left(t-T_{\mathrm{s}}-365(k-1)\right)\right)$ in $T_{\mathrm{s}}+365(k-1)<t<365 k$ for $k=1,2, \ldots$. The numerical results for $0 \leq t \leq 1825$ are shown in the figures.

$\left(P_{2}^{\mathrm{e}}, X_{2}^{\mathrm{e}}\right),\left(P_{2}^{\mathrm{h}}, X_{2}^{\mathrm{h}}\right)$, and $\left(P_{2}^{\mathrm{b}}, X_{2}^{\mathrm{b}}\right)$. Eventually, we have all solutions for $k=1,2, \ldots$ by an inductive argument. In order to study the total dynamics of the two state variables, we consider $\left(\bar{P}_{k}(t-\right.$ $\left.365(k-1)), \bar{X}_{k}(t-365(k-1))\right)$, in 365(k-1) $<t<T_{\mathrm{s}}+365(k-1)$ and $\left(P_{k}\left(t-T_{\mathrm{s}}-365(k-1)\right), X_{k}(t-\right.$ $\left.\left.T_{\mathrm{S}}-365(k-1)\right)\right)$ in $T_{\mathrm{S}}+365(k-1)<t<365 k$ for $k=1,2, \ldots$, where $\left(\bar{P}_{k}, \bar{X}_{k}\right)$ is a spatial average of $\left(P_{k}^{\mathrm{e}}, X_{k}^{\mathrm{e}}\right),\left(P_{k}^{\mathrm{h}}, X_{k}^{\mathrm{h}}\right)$, and $\left(P_{k}^{\mathrm{b}}, X_{k}^{\mathrm{b}}\right)$, defined by

$$
\begin{aligned}
& \bar{P}_{k}(t)=\frac{1}{Z}\left(Z_{\mathrm{e}} P_{k}^{\mathrm{e}}(t)+\int_{0}^{Z_{\mathrm{h}}} P_{k}^{\mathrm{h}}(z, t) \mathrm{d} z+Z_{\mathrm{b}} P_{k}^{\mathrm{b}}(t)\right), \\
& \bar{X}_{k}(t)=\frac{1}{Z}\left(Z_{\mathrm{e}} X_{k}^{\mathrm{e}}(t)+\int_{0}^{Z_{\mathrm{h}}} X_{k}^{\mathrm{h}}(z, t) \mathrm{d} z+Z_{\mathrm{b}} X_{k}^{\mathrm{b}}(t)\right) .
\end{aligned}
$$

Here we show an example of the total dynamics generated by the system (2.1)-(2.7) (See Figure 1).

\section{Reduction of model}


Our mathematical model (2.1) - (2.7) is mode realistic than those proposed in the previous studies [2] and [7] because it includes interaction between phytoplankton and phosphorus with influences of temporally dynamic features in ecology and physico-chemical environments, which incorporates 1-dimensional vertical structure of lake ecosystem and seasonal periodic cycle of water mixing. As shown in Figure 1, the solution of the system (2.1)-(2.7) changes periodically in time. Generally speaking, it is difficult to study periodic solutions in ordinary differential equations analytically. Then we formally reduce the system (2.1)-(2.7) to establish the existence and stability of periodic solutions analytically in a reduced system.

We first assume that the solutions $P_{k}^{\mathrm{e}}(t), X_{k}^{\mathrm{e}}(t), X_{k}^{\mathrm{b}}(t)$ of (2.1) and (2.3) rapidly converge to steady states. More precisely, we suppose that we can omit $\mathrm{d} P_{k}^{\mathrm{e}} / \mathrm{d} t, \mathrm{~d} X_{k}^{\mathrm{e}} / \mathrm{d} t$, and $\mathrm{d} X_{k}^{\mathrm{b}} / \mathrm{d} t$ in (2.1) and (2.3). From this assumption, we can set $\left(P_{k}^{\mathrm{e}}(t), X_{k}^{\mathrm{e}}(t)\right)=\left(P_{*}^{\mathrm{e}}, X_{*}^{\mathrm{e}}\right)$ for any $0<t<T_{\mathrm{s}}$ $(k=1,2, \ldots)$, which is a stationary solution of $(2.1)$ given by

$$
P_{*}^{\mathrm{e}}=\frac{l Z_{\mathrm{e}}^{\gamma-2}\left(\beta_{\mathrm{e}} Z_{\mathrm{e}}+s_{\mathrm{e}}\right)}{\alpha\left(s_{\mathrm{e}}+\beta_{\mathrm{e}} Z_{\mathrm{e}}\left(1-c_{1} c_{2}\right)\right)}, \quad X_{*}^{\mathrm{e}}=\frac{c_{2} l}{s_{\mathrm{e}}+\beta_{\mathrm{e}} Z_{\mathrm{e}}\left(1-c_{1} c_{2}\right)} .
$$

Note that $\left(P_{*}^{\mathrm{e}}, X_{*}^{\mathrm{e}}\right)$ is determined independently on $k$. Similarly, $X_{k}^{\mathrm{b}}(t)$ can be explicitly given by

$$
X_{k}^{\mathrm{b}}(t)=\frac{s_{\mathrm{h}}}{\beta_{\mathrm{b}} Z_{\mathrm{b}}+s_{\mathrm{b}}} X_{k}^{\mathrm{h}}\left(Z_{\mathrm{h}}, t\right)
$$

Since (2.5) is an autonomous linear differential system, the solution $\left(P_{k}(t), X_{k}(t)\right)$ of (2.5) can be represented by

$$
\left(P_{k}(t), X_{k}(t)\right)=\mathbf{c}_{0}(l)+e^{\lambda_{1} t} \mathbf{c}_{1}\left(l, P_{k}(0), X_{k}(0)\right)+e^{\lambda_{2} t} \mathbf{c}_{2}\left(l, P_{k}(0), X_{k}(0)\right),
$$

where $\mathbf{c}_{0}(l), \mathbf{c}_{i}(l, P, X)(i=1,2)$ are vectors linearly dependent on $l, P, X$. The exponents $\lambda_{1}, \lambda_{2}$ are given by

$$
\begin{aligned}
& \lambda_{1}=-\frac{1}{2}\left(\frac{\alpha}{Z^{\gamma-1}}+\beta+\frac{s_{\mathrm{b}}}{Z}-\omega\right), \quad \lambda_{2}=-\frac{1}{2}\left(\frac{\alpha}{Z^{\gamma-1}}+\beta+\frac{s_{\mathrm{b}}}{Z}+\omega\right), \\
& \omega=\sqrt{\left(\frac{\alpha}{Z^{\gamma-1}}\right)^{2}+4 c_{1} c_{2} \frac{\alpha}{Z^{\gamma-1}} \beta-2 \frac{\alpha}{Z^{\gamma-1}}\left(\beta+\frac{s_{\mathrm{b}}}{Z}\right)+\left(\beta+\frac{s_{\mathrm{b}}}{Z}\right)^{2}} .
\end{aligned}
$$

It is obvious that $\lambda_{2}<\lambda_{1}<0$ holds under the assumptions of $0 \leq c_{1} \leq 1$ and $0 \leq c_{2} \leq 1$. Since $e^{\lambda_{2} t}$ decays rapidly, we formally omit $e^{\lambda_{2} t} \mathbf{c}_{2}\left(l, P_{k}(0), X_{k}(0)\right)$ in (2.8) and assume that the solution $\left(P_{k}(t), X_{k}(t)\right)$ of (2.5) can be approximated by $\left(P_{k}(t), X_{k}(t)\right)=\mathbf{c}_{0}(l)+e^{\lambda_{1} t} \mathbf{c}_{1}\left(l, P_{k}(0), X_{k}(0)\right)$. Actually, we substitute all parameter values into $\lambda_{1}$ and $\lambda_{2}$ in Table 1 , and then have $\left(\lambda_{1}, \lambda_{2}\right)=$ $(-0.026,-1.1)$ for Set I and $\left(\lambda_{1}, \lambda_{2}\right)=(-0.0048,-0.16)$ for Set II, respectively. Hence we can compute

$$
\left(\begin{array}{c}
P_{k}\left(T_{\mathrm{w}}\right) \\
X_{k}\left(T_{\mathrm{w}}\right)
\end{array}\right)=\mathbf{v}_{\mathrm{w}}+M_{\mathrm{w}}\left(\begin{array}{c}
P_{k}(0) \\
X_{k}(0)
\end{array}\right)
$$


where $\mathbf{v}_{\mathrm{w}}={ }^{t}\left(v_{\mathrm{w} 1}, v_{\mathrm{w} 2}\right)$ and $M_{\mathrm{w}}=\left(\begin{array}{cc}m_{\mathrm{w} 11} & m_{\mathrm{w} 12} \\ m_{\mathrm{w} 21} & m_{\mathrm{w} 22}\end{array}\right)$ are defined by

$$
\begin{aligned}
\left(\begin{array}{l}
v_{\mathrm{w} 1} \\
v_{\mathrm{w} 2}
\end{array}\right) & =\left(\begin{array}{c}
\frac{l Z^{\gamma-2}\left(\beta Z+s_{\mathrm{b}}\right)}{\alpha\left(s_{\mathrm{b}}+\beta Z\left(1-c_{1} c_{2}\right)\right)}\left(1+\frac{\lambda_{2}}{\omega} e^{\lambda_{1} T_{\mathrm{w}}}\right)+\frac{l}{\omega} e^{\lambda_{1} T_{\mathrm{w}}} \\
\frac{c_{2} l}{s_{\mathrm{b}}+\beta Z\left(1-c_{1} c_{2}\right)}\left(1+\frac{\lambda_{2}}{\omega} e^{\lambda_{1} T_{\mathrm{w}}}\right)
\end{array}\right), \\
\left(\begin{array}{ll}
m_{\mathrm{w} 11} & m_{\mathrm{w} 12} \\
m_{\mathrm{w} 21} & m_{\mathrm{w} 22}
\end{array}\right) & =e^{\lambda_{1} T_{\mathrm{w}}}\left(\begin{array}{cc}
\frac{1}{2 \omega}\left(\beta+\frac{s_{\mathrm{b}}}{Z}+\omega-\frac{\alpha}{Z^{\gamma-1}}\right) & \frac{1}{2 \omega}\left(-\beta-\frac{s_{\mathrm{b}}}{Z}+\omega+\frac{\alpha}{Z^{\gamma-1}}\right) \\
c_{2} \frac{\alpha}{\omega Z^{\gamma-1}} & \frac{c_{1}}{2 \omega}
\end{array} .\right.
\end{aligned}
$$

Under these assumptions, we can represent all the other solutions explicitly by an inductive argument. In the following, we hypothesize an extra condition $s_{\mathrm{h}} T_{\mathrm{s}}>Z_{\mathrm{h}}$. Then the solutions $X_{k+1}^{\mathrm{h}}, X_{k+1}^{\mathrm{b}}, u_{k+1}$ are represented as follows;

$$
\begin{aligned}
X_{k+1}^{\mathrm{h}}(z, t) & = \begin{cases}e^{-\beta_{\mathrm{h}} t} X_{k}\left(T_{\mathrm{w}}\right), & 0 \leq t \leq T_{\mathrm{s}}, s_{\mathrm{h}} t \leq z \leq Z_{\mathrm{h}}, \\
e^{-\frac{\beta_{\mathrm{h}}}{s_{\mathrm{h}}} X_{*}^{\mathrm{e}},} \quad 0 \leq t \leq T_{\mathrm{s}}, 0 \leq z<s_{\mathrm{h}} t,\end{cases} \\
X_{k+1}^{\mathrm{b}}(t) & = \begin{cases}\frac{s_{\mathrm{h}}}{\beta_{\mathrm{b}} Z_{\mathrm{b}}+s_{\mathrm{b}}} e^{-\beta_{\mathrm{h}} t} X_{k}\left(T_{\mathrm{w}}\right), & 0 \leq t \leq \frac{Z_{\mathrm{h}}}{s_{\mathrm{h}}}, \\
\frac{s_{\mathrm{h}}}{\beta_{\mathrm{b}} Z_{\mathrm{b}}+s_{\mathrm{b}}} e^{-\frac{\beta_{\mathrm{h}} Z_{\mathrm{h}}}{s_{\mathrm{h}}}} X_{*}^{\mathrm{e}}, & \frac{Z_{\mathrm{h}}}{s_{\mathrm{h}}}<t \leq T_{\mathrm{s}},\end{cases} \\
u_{k+1}(t) & = \begin{cases}u_{0} \exp \left(-\frac{\left(1-e^{-\beta_{\mathrm{h}} t}\right) s_{\mathrm{h}} X_{k}\left(T_{\mathrm{w}}\right) \kappa}{\beta_{\mathrm{h}}\left(\beta_{\mathrm{b}} Z_{\mathrm{b}}+s_{\mathrm{b}}\right)}\right), & 0 \leq t \leq \frac{Z_{\mathrm{h}}}{s_{\mathrm{h}}}, \\
u_{0} \exp \left(-\frac{\beta_{\mathrm{h}} e^{-\frac{\beta_{\mathrm{h}} Z_{\mathrm{h}}}{s_{\mathrm{h}}}}\left(s_{\mathrm{h}} t-Z_{\mathrm{h}}\right) X_{*}^{\mathrm{e}}+\left(1-e^{-\frac{\beta_{\mathrm{h}} Z_{\mathrm{h}}}{s_{\mathrm{h}}}}\right) s_{\mathrm{h}} X_{k}\left(T_{\mathrm{w}}\right)}{\beta_{\mathrm{h}}\left(\beta_{\mathrm{b}} Z_{\mathrm{b}}+s_{\mathrm{b}}\right)} \kappa\right), & \frac{Z_{\mathrm{h}}}{s_{\mathrm{h}}}<t \leq T_{\mathrm{s}} .\end{cases}
\end{aligned}
$$

Since $u_{k+1}(t)$ decreases monotonically in time, $f\left(u_{k+1}(t)\right)$ jumps at the time of $u_{k+1}(t)=$ $\theta$ if it happens. Based on the above representation of $u_{k+1}(t)$, we define $T_{1}=T_{1}(x)$ and $T_{2}=$ $T_{2}(x)$ by

$$
\begin{aligned}
& T_{1}(x)=\frac{1}{\beta_{\mathrm{h}}} \log \left(\frac{s_{\mathrm{h}} x \kappa}{s_{\mathrm{h}} x \kappa-\beta_{\mathrm{h}}\left(\beta_{\mathrm{b}} Z_{\mathrm{b}}+s_{\mathrm{b}}\right) \log \left(\frac{u_{0}}{\theta}\right)}\right), \\
& T_{2}(x)=\frac{\left(1-e^{\frac{\beta_{\mathrm{h}} Z_{\mathrm{h}}}{s_{\mathrm{h}}}}\right) s_{\mathrm{h}} x \kappa+\beta_{\mathrm{h}} Z_{\mathrm{h}} X_{*}^{\mathrm{e}} \kappa+\beta_{\mathrm{h}} e^{\frac{\beta_{\mathrm{h}} Z_{\mathrm{h}}}{s_{\mathrm{h}}}}\left(\beta_{\mathrm{b}} Z_{\mathrm{b}}+s_{\mathrm{b}}\right) \log \left(\frac{u_{0}}{\theta}\right)}{\beta_{\mathrm{h}} s_{\mathrm{h}} X_{*}^{\mathrm{e}} \kappa} .
\end{aligned}
$$

If $X_{k}\left(T_{\mathrm{W}}\right)$ satisfies $T_{1}\left(X_{k}\left(T_{\mathrm{W}}\right)\right)<Z_{\mathrm{h}} / s_{\mathrm{h}}$, we have $u_{k+1}(t)>\theta$ for $0<t<T_{1}\left(X_{k}\left(T_{\mathrm{W}}\right)\right)$ and $u_{k+1}(t)<$ $\theta$ for $T_{1}\left(X_{k}\left(T_{\mathrm{W}}\right)\right)<t<T_{\mathrm{s}}$ so that $f\left(u_{k+1}(t)\right)=R$ for $T_{1}\left(X_{k}\left(T_{\mathrm{W}}\right)\right)<t<T_{\mathrm{s}}$. Similarly, if $X_{k}\left(T_{\mathrm{W}}\right)$ satisfies $Z_{\mathrm{h}} / s_{\mathrm{h}}<T_{2}\left(X_{k}\left(T_{\mathrm{w}}\right)\right)<T_{\mathrm{s}}$, we have $u_{k+1}(t)>\theta$ for $0<t<T_{2}\left(X_{k}\left(T_{\mathrm{W}}\right)\right)$ and $u_{k+1}(t)<\theta$ for $T_{2}\left(X_{k}\left(T_{\mathrm{W}}\right)\right)<t<T_{\mathrm{s}}$ so that $f\left(u_{k+1}(t)\right)=R$ for $T_{2}\left(X_{k}\left(T_{\mathrm{W}}\right)\right)<t<T_{\mathrm{s}}$. 
In order to describe the properties of $T_{1}(x)$ and $T_{2}(x)$, we introduce $x_{0}, x_{1}, x_{2}, x_{c}$ defined by

$$
\begin{gathered}
x_{0}=\frac{\beta_{\mathrm{h}}\left(\left(Z_{\mathrm{h}}-s_{\mathrm{h}} T_{\mathrm{s}}\right) X_{*}^{\mathrm{e}} \kappa+e^{\frac{\beta_{\mathrm{h}} Z_{\mathrm{h}}}{s_{\mathrm{h}}}}\left(\beta_{\mathrm{b}} Z_{\mathrm{b}}+s_{\mathrm{b}}\right) \log \left(\frac{u_{0}}{\theta}\right)\right)}{\left(e^{\frac{\beta_{\mathrm{h}} Z_{\mathrm{h}}}{s_{\mathrm{h}}}}-1\right) s_{\mathrm{h}} \kappa}, \quad x_{1}=\frac{\beta_{\mathrm{h}}\left(\beta_{\mathrm{b}} Z_{\mathrm{b}}+s_{\mathrm{b}}\right) \log \left(\frac{u_{0}}{\theta}\right)}{s_{\mathrm{h}} \kappa}, \\
x_{2}=\frac{\beta_{\mathrm{h}}\left(Z_{\mathrm{h}} X_{*}^{\mathrm{e}} \kappa+e^{\frac{\beta_{\mathrm{h}} Z_{\mathrm{h}}}{s_{\mathrm{h}}}}\left(\beta_{\mathrm{b}} Z_{\mathrm{b}}+s_{\mathrm{b}}\right) \log \left(\frac{u_{0}}{\theta}\right)\right)}{\left(e^{\frac{\beta_{\mathrm{h}} Z_{\mathrm{h}}}{s_{\mathrm{h}}}}-1\right) s_{\mathrm{h}} \kappa}, \quad x_{c}=\frac{\beta_{\mathrm{h}} e^{\frac{\beta_{\mathrm{h}} Z_{\mathrm{h}}}{s_{\mathrm{h}}}}\left(\beta_{\mathrm{b}} Z_{\mathrm{b}}+s_{\mathrm{b}}\right) \log \left(\frac{u_{0}}{\theta}\right)}{\left(e^{\frac{\beta_{\mathrm{h}} Z_{\mathrm{h}}}{s_{\mathrm{h}}}}-1\right) s_{\mathrm{h}} \kappa} .
\end{gathered}
$$

We note that $x_{1}<x_{c}<x_{2}$ and $x_{0}<x_{c}$ under the assumptions of $s_{\mathrm{h}} T_{\mathrm{s}}>Z_{\mathrm{h}}$ and $u_{0}>\theta$. It is easy to check all properties described in the next proposition. Hence we omit the details of the proof.

Proposition 2.1. The function $T_{1}$ is positive, monotonically decreasing and concave in $x_{1}<x$. Similarly, $T_{2}$ is positive and monotonically decreasing in $x<x_{2}$. In addition, it holds that

$$
\lim _{x \rightarrow \infty} T_{1}(x)=0, \quad T_{2}\left(x_{2}\right)=0, \quad T_{1}\left(x_{c}\right)=T_{2}\left(x_{c}\right)=\frac{Z_{\mathrm{h}}}{s_{\mathrm{h}}}, \quad T_{2}\left(x_{0}\right)=T_{\mathrm{s}} .
$$

By the same argument as $X_{k+1}^{\mathrm{h}}$ and $X_{k+1}^{\mathrm{b}}$, we can explicitly define $P_{k+1}^{\mathrm{h}}(z, t)$ and $P_{k+1}^{\mathrm{b}}(t)$ though we do not describe their complicated forms here. As a result, we obtain

$$
\begin{aligned}
& \frac{1}{Z_{\mathrm{h}}} \int_{0}^{Z_{\mathrm{h}}} P_{k+1}^{\mathrm{h}}\left(z, T_{\mathrm{s}}\right) d z=P_{k}\left(T_{\mathrm{W}}\right)+c_{1}\left(1-\frac{s_{\mathrm{h}}}{Z_{\mathrm{h}} \beta_{\mathrm{h}}}\left(1-e^{-\frac{\beta_{\mathrm{h}} Z_{\mathrm{h}}}{s_{\mathrm{h}}}}\right)\right) X_{k}\left(T_{\mathrm{w}}\right) \\
&+c_{1}\left(\frac{s_{\mathrm{h}}}{Z_{\mathrm{h}}}\left(T_{\mathrm{s}}-\frac{1}{\beta_{\mathrm{h}}}\right)\left(1-e^{-\frac{\beta_{\mathrm{h}} Z_{\mathrm{h}}}{s_{\mathrm{h}}}}\right)+e^{-\frac{\beta_{\mathrm{h}} Z_{\mathrm{h}}}{s_{\mathrm{h}}}}\right) X_{*}^{\mathrm{e}}, \\
& P_{1}^{\mathrm{b}}\left(T_{\mathrm{s}}\right)=P_{k}\left(T_{\mathrm{w}}\right)+\frac{R}{Z_{\mathrm{b}}} T\left(X_{k}\left(T_{\mathrm{W}}\right)\right) \\
&+c_{1} \frac{\beta_{\mathrm{b}}\left(s_{\mathrm{h}} T_{\mathrm{s}}-Z_{\mathrm{h}}\right)}{\beta_{\mathrm{b}} Z_{\mathrm{b}}+s_{\mathrm{b}}} e^{-\frac{\beta_{\mathrm{h}} Z_{\mathrm{h}}}{s_{\mathrm{h}}}} X_{*}^{\mathrm{e}}+c_{1} \frac{\beta_{\mathrm{b}} s_{\mathrm{h}}}{\beta_{\mathrm{h}}\left(\beta_{\mathrm{b}} Z_{\mathrm{b}}+s_{\mathrm{b}}\right)}\left(1-e^{-\frac{\beta_{\mathrm{h}} Z_{\mathrm{h}}}{s_{\mathrm{h}}}}\right) X_{k}\left(T_{\mathrm{W}}\right),
\end{aligned}
$$

where $T(x)$ is defined by

$$
T(x)= \begin{cases}0, & 0 \leq x<\max \left\{0, x_{0}\right\} \\ T_{\mathrm{s}}-T_{2}(x), & \max \left\{0, x_{0}\right\} \leq x<x_{c} \\ T_{\mathrm{s}}-T_{1}(x), & x_{c} \leq x\end{cases}
$$

From Proposition 2.1, we see that $T(x)$ is a nonnegative, monotonically increasing, continuous function in $0 \leq x$, and smooth except for $x=\max \left\{0, x_{0}\right\}, x_{c}$. 
Finally we compute the initial condition for $\left(P_{k+1}(0), X_{k+1}(0)\right)$ by $(2.7)$ such as

$$
\left(\begin{array}{c}
P_{k+1}(0) \\
X_{k+1}(0)
\end{array}\right)=\mathbf{v}_{\mathrm{s}}+M_{\mathrm{s}}\left(\begin{array}{c}
P_{k}\left(T_{\mathrm{w}}\right) \\
X_{k}\left(T_{\mathrm{w}}\right)
\end{array}\right)+\frac{R}{Z} T\left(X_{k}\left(T_{\mathrm{w}}\right)\right)\left(\begin{array}{l}
1 \\
0
\end{array}\right),
$$

where $\mathbf{v}_{\mathrm{s}}={ }^{t}\left(v_{\mathrm{s} 1}, v_{\mathrm{s} 2}\right)$ and $M_{\mathrm{s}}=\left(\begin{array}{cc}m_{\mathrm{s} 11} & m_{\mathrm{s} 12} \\ 0 & 0\end{array}\right)$ are defined by

$$
\begin{gathered}
\left(\begin{array}{c}
v_{\mathrm{s} 1} \\
v_{\mathrm{s} 2}
\end{array}\right)=\frac{1}{Z}\left(\begin{array}{c}
Z_{\mathrm{e}} P_{*}^{\mathrm{e}}+c_{1}\left(s_{\mathrm{h}}\left(T_{\mathrm{s}}-\frac{1}{\beta_{\mathrm{h}}}\left(1-e^{-\frac{\beta_{\mathrm{h}} Z_{\mathrm{h}}}{s_{\mathrm{h}}}}\right)\right)-\frac{s_{\mathrm{b}}\left(s_{\mathrm{h}} T_{\mathrm{s}}-Z_{\mathrm{h}}\right)}{\beta_{\mathrm{b}} Z_{\mathrm{b}}+s_{\mathrm{b}}} e^{-\frac{\beta_{\mathrm{h}} Z_{\mathrm{h}}}{s_{\mathrm{h}}}}\right) X_{*}^{\mathrm{e}} \\
\left(Z_{\mathrm{e}}+\frac{s_{\mathrm{h}}}{\beta_{\mathrm{h}}}\left(1-e^{-\frac{\beta_{\mathrm{h}} Z_{\mathrm{h}}}{s_{\mathrm{h}}}}\right)+\frac{Z_{\mathrm{b}} s_{\mathrm{h}}}{\beta_{\mathrm{b}} Z_{\mathrm{b}}+s_{\mathrm{b}}} e^{-\frac{\beta_{\mathrm{h}} Z_{\mathrm{h}}}{s_{\mathrm{h}}}}\right) X_{*}^{\mathrm{e}}
\end{array}\right), \\
m_{\mathrm{s} 11}=\frac{Z_{\mathrm{b}}+Z_{\mathrm{h}}}{Z}, \quad m_{\mathrm{s} 12}=c_{1}\left(\frac{Z_{\mathrm{h}}}{Z}-\frac{\left(1-e^{-\frac{\beta_{\mathrm{h}} Z_{\mathrm{h}}}{s_{\mathrm{h}}}}\right) s_{\mathrm{b}} s_{\mathrm{h}}}{\beta_{\mathrm{h}} Z\left(\beta_{\mathrm{b}} Z_{\mathrm{b}}+s_{\mathrm{b}}\right)}\right) .
\end{gathered}
$$

Combining (2.9) and (2.10), we have

$$
\left(\begin{array}{c}
P_{k+1}\left(T_{\mathrm{W}}\right) \\
X_{k+1}\left(T_{\mathrm{w}}\right)
\end{array}\right)=M\left(\begin{array}{c}
P_{k}\left(T_{\mathrm{W}}\right) \\
X_{k}\left(T_{\mathrm{w}}\right)
\end{array}\right)+\mathbf{v}_{1}+\frac{R}{Z} T\left(X_{k}\left(T_{\mathrm{w}}\right)\right) \mathbf{v}_{2},
$$

for all $k \geq 0$, where $M=M_{\mathrm{W}} M_{\mathrm{s}}, \mathbf{v}_{1}=\mathbf{v}_{\mathrm{w}}+M_{\mathrm{w}} \mathbf{v}_{\mathrm{s}}$ and $\mathbf{v}_{2}={ }^{t}\left(m_{\mathrm{w} 11}, m_{\mathrm{w} 21}\right)$. Instead of solving the system (2.1) - (2.7) directly, we treat the discrete dynamical system (2.11) with two state variable $\left\{\left(P_{k}\left(T_{\mathrm{w}}\right), X_{k}\left(T_{\mathrm{w}}\right)\right)\right\}_{k=0}^{\infty}$.

\section{Results}

We first see that the system (2.1) - (2.7) can generate two kinds of periodic solutions for the same parameters dependently on initial values, which means that our model has a hysteresis structure (Figure 1). The results seem to correspond to those obtained in [5]. However it is difficult to prove the existence and stability of periodic solutions analytically in our model. Instead of the continuous model (2.1)-(2.7), we consider a discrete model (2.11) and prove the existence and stability of fixed points. First we consider the existence of a fixed point of (2.11). If there exists a fixed point $(P, X)$ in $(2.11),(P, X)$ satisfies

$$
(I-M)\left(\begin{array}{c}
P \\
X
\end{array}\right)=\mathbf{v}_{1}+\frac{R}{Z} T(X) \mathbf{v}_{2},
$$

where $I$ is the identity matrix on $\mathbb{R}^{2}$. If $I-M$ is invertible, we have

$$
\left(\begin{array}{l}
P \\
X
\end{array}\right)=(I-M)^{-1} \mathbf{v}_{1}+\frac{R}{Z} T(X)(I-M)^{-1} \mathbf{v}_{2} .
$$




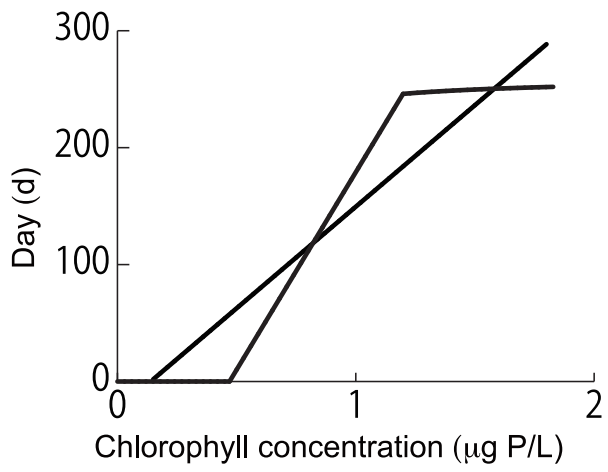

Figure 2: Graphs of $T=\left(X-x_{*}\right) / r_{x}$ and $T=T(X)$ in (3.2). In the figure, three fixed points of (3.2) is realized by the intersection points of the two graphs. We set $l=0.3$ and use the parameters of Set I in Table 1 to generate this figure.

Here we denote

$$
\left(\begin{array}{c}
p_{*} \\
x_{*}
\end{array}\right)=(I-M)^{-1} \mathbf{v}_{1}, \quad\left(\begin{array}{c}
r_{p} \\
r_{x}
\end{array}\right)=\frac{R}{Z}(I-M)^{-1} \mathbf{v}_{2}=\frac{R}{Z} \frac{1}{\operatorname{det}(I-M)} \mathbf{v}_{2} .
$$

Since the right-hand side of (3.1) does not have the variable $P$, it suffices to find a fixed point of $X=x_{*}+r_{x} T(X)$, or equivalently,

$$
\frac{1}{r_{x}} X-\frac{x_{*}}{r_{x}}=T(X)
$$

and then set $P=p_{*}+r_{p} T(X)$ for a fixed point $X$ of (3.2) in order to prove the existence of a fixed point of (2.11). Note that there is only one fixed point given by $X=x_{*}$ in the case of $r_{x}=0$.

The stability of the fixed point $(P, X)$ is determined by the linearized eigenvalue problem

$$
\lambda\left(\begin{array}{c}
\phi \\
\psi
\end{array}\right)=M\left(\begin{array}{c}
\phi \\
\psi
\end{array}\right)+\frac{R}{Z} T^{\prime}(X) \psi \mathbf{v}_{2}=M_{\mathrm{W}}\left(M_{\mathrm{s}}+\frac{R}{Z} T^{\prime}(X) J\right)\left(\begin{array}{c}
\phi \\
\psi
\end{array}\right)
$$

where $J=\left(\begin{array}{ll}0 & 1 \\ 0 & 0\end{array}\right)$. Here we assume that the fixed point $X$ is not equal to $x_{c}, x_{0}$ where $T(X)$ is not differentiable. Since the $(2,1)$ and $(2,2)$ entries of both $M_{\mathrm{s}}$ and $J$ are 0 , the eigenvalue problem above has 0 eigenvalue. The other eigenvalue $\lambda$ can be explicitly given by

$$
\lambda=\operatorname{tr}(M)+m_{\mathrm{w} 21} \frac{R}{Z} T^{\prime}(X)=1-\operatorname{det}(I-M)+m_{\mathrm{w} 21} \frac{R}{Z} T^{\prime}(X) .
$$

Since $T^{\prime}(X)$ is nonnegative for any $X \neq x_{0}, x_{c}$, the fixed point becomes unstable in the case of $\operatorname{det}(I-M)<0$. Therefore the following lemma holds. 
Lemma 3.2. Suppose that there is a fixed point $(P, X)$ of (3.1) with nonnegative $X \neq x_{0}, x_{c}$. If $\operatorname{det}(I-M)$ is negative, $(P, X)$ must be unstable in (2.11).

From Lemma 3.2, the bistability in (2.11) may occur only in the case of $\operatorname{det}(I-M)>0$. In this case, $r_{x}$ is positive because of its explicit expression. Actually, if we apply the parameters of Sets I and II in Table 1, we have $\operatorname{det}(I-M)>0$. In addition, since the eigenvalue $\lambda$ given in (3.4) is rewritten as

$$
\lambda=1+m_{\mathrm{w} 21} \frac{R}{Z}\left(T^{\prime}(X)-\frac{1}{r_{x}}\right),
$$

the stability of the fixed point of (3.2) can be determined by the difference between two slopes of the line $T=\left(X-x_{*}\right) / r_{x}$ and $T=T(X)$ at an intersection point.

The equation (3.2) can posses a fixed point for each $T(x)=0, T_{\mathrm{S}}-T_{1}(x), T_{\mathrm{S}}-T_{2}(x)$. The solution of $\left(X-x_{*}\right) / r_{x}=0$ is trivially given by $X=x_{*}$. In this case we denote the solution by $\bar{X}_{0}(l)=x_{*}$ and emphasis the $l$-dependence of the solution. Here we note that $x_{*}$ is equal to 0 at $l=0$ and increases linearly as $l$ increases under the assumptions of $\operatorname{det}(I-M)>0$ and $s_{\mathrm{h}} T_{\mathrm{S}}>Z_{\mathrm{h}}$. Similarly, $x_{0}$ is positive at $l=0$ and decreases linearly as $l$ increases so that it achieves 0 for some $l$. Hence there is some $l_{0}>0$ such that $x_{*}=x_{0}$ at $l=l_{0}$. Note that $\bar{X}_{0}(l)$ is positive in $0<l \leq l_{0}$. Similarly, we denote the solution of $\left(X-x_{*}\right) / r_{x}=T_{\mathrm{s}}-T_{2}(X)$ by $\bar{X}_{2}(l)$. From the definition of $T(x), \bar{X}_{2}(l)$ is a fixed point of (3.2) if it satisfies $\max \left\{0, x_{0}\right\} \leq \bar{X}_{2}(l) \leq x_{c}$. Since $x_{*}$ depends on $l$ linearly and $T_{\mathrm{S}}-T_{2}\left(x_{c}\right)=Z_{\mathrm{h}} / s_{\mathrm{S}}$, there is $l=l_{c}$ such that $\left(x_{c}-x_{*}\right) / r_{x}=$ $Z_{\mathrm{h}} / s_{\mathrm{s}}$. Then we see $\bar{X}_{2}\left(l_{c}\right)=x_{c}$. Note that $l_{c}$ can be negative for some parameters. Finally, we consider the case of $T(x)=T_{\mathrm{s}}-T_{1}(x)$. Since $T_{1}(x)$ is a logarithmic function, there are $l_{t}$ and $x_{t}>0$ such that $\left(x-x_{*}\right) / r_{x}$ is tangent to $T_{\mathrm{s}}-T_{2}(x)$ at $x=x_{t}$ for $l=l_{t}$. In other words, it holds that

$$
\frac{1}{r_{x}}\left(x_{t}-x_{*}\right)=T_{\mathrm{s}}-T_{1}\left(x_{t}\right), \quad \frac{1}{r_{x}}=-T_{1}^{\prime}\left(x_{t}\right)
$$

for $l=l_{t}$. Then the equation $\left(X-x_{*}\right) / r_{x}=T_{\mathrm{S}}-T_{2}(X)$ has exactly two solutions for $l>l_{t}$, denoted by $\bar{X}_{1}^{\text {low }}(l)$ and $\bar{X}_{1}^{\text {high }}(l)$ with $\bar{X}_{1}^{\text {low }}(l)<\bar{X}_{1}^{\text {high }}(l)$, and no solution for $l<l_{t}$. Under the assumption, $l_{t}$ must be less than $l_{c}$.

Due to the representation formula for the eigenvalue defined in (3.4) and the fact that the fixed points of (3.2) is categorized into four types, we can detect bifurcation points in (2.11). Therefore we have the following proposition.

Proposition 3.3. The number and stability of fixed points in (2.11) change only at $l=l_{0}, l_{c}, l_{t}$.

Thanks to $l_{0}>0, l_{t}<l_{c}$ and Proposition 3.3, we can categorize the bifurcation diagrams of (2.11) in a generic sense. The bifurcation diagrams of (2.11) are divided into eight types; $x_{t}<x_{c}, 0<l_{0}<l_{c}$ (Type I); $x_{t}<x_{c}, 0<l_{c}<l_{0}$ (Type II); $l_{t}<0, l_{c}<0$ (Type III); $x_{c}<x_{t}, 0<$ $l_{0}<l_{t}<l_{c}$ (Type IV); $x_{c}<x_{t}, 0<l_{t}<l_{0}<l_{c}$ (Type V); $x_{c}<x_{t}, 0<l_{t}<l_{c}<l_{0}$ (Type VI); 


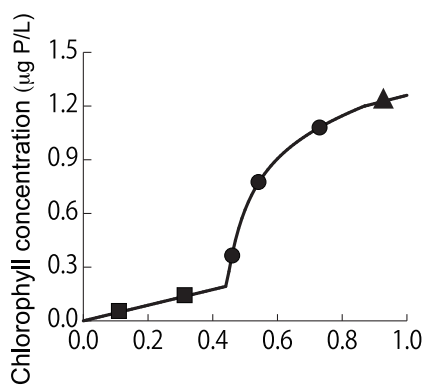

(a) Type I

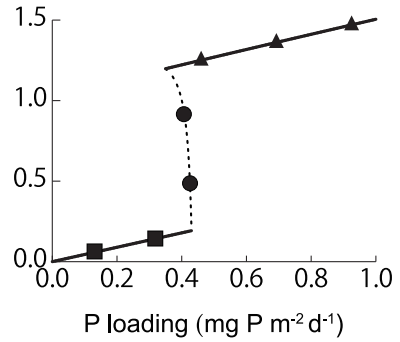

(b) Type II

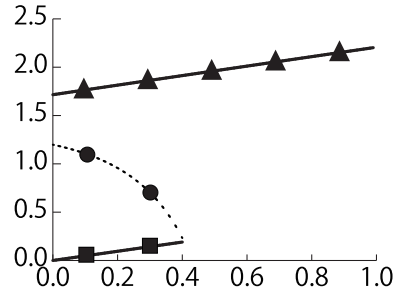

(c) Type III

Figure 3: Response types I-III of (2.11). The horizontal and vertical axes represent the bifurcation parameter $l$ and the fixed points of (3.2). The lines with square, disk, and triangle symbols correspond to the fixed points $\bar{X}_{0}(l), \bar{X}_{2}(l)$, and $\bar{X}_{1}^{\text {high }}(l)$, respectively. The solid (dashed) lines mean that each fixed point is stable (unstable) in (2.11). The parameters of Set I in Table 1 are used except for $T_{\mathrm{s}}$. (a) $T_{\mathrm{s}}=244$; (b) $T_{\mathrm{s}}=251$; (c) $T_{\mathrm{s}}=265$.

$x_{c}<x_{t}, l_{t}<0,0<l_{c}<l_{0}$ (Type VII); $x_{c}<x_{t}, l_{t}<0,0<l_{0}<l_{c}$ (Type VIII) (see Figures 3-5). Hereafter we refer to these as the response types of the lake.

Now we are in a position to show the bifurcation diagrams of (2.11) for the parameter $l$. In Figure 3, no hysteresis structure appears in Type I (reversible) while it emerges in Type II for $l_{c}<l<l_{0}$ (hysteretic) and Type III for $l<l_{0}$ (irreversible). These three bifurcation diagrams are similar to those in Fig.1 in [5]. We see that the number of fixed points of (3.2) is 1 or 3 except for the critical points $l=l_{0}, l_{c}, l_{t}$. In three results shown in Figure 3, we only change $T_{\mathrm{s}}$. The difference of $T_{\mathrm{s}}$ between Types I and III is only 21 [d]. Hence we conclude that the period of summer $T_{\mathrm{s}}$ strongly affects the hysteresis structure.

In Figures 4,5 , the fixed point $\bar{X}_{1}^{\text {low }}$ appears, which is always unstable in all types. All results in Figure 4 correspond to the hysteretic type. In three results shown in Figure 4, we only change $T_{\mathrm{s}}$. The difference of $T_{\mathrm{s}}$ between Types I and III is only 10 [d]. On the other hand, two bifurcation diagrams in Figure 5 are categorized into an irreversible type. The bifurcation diagrams in Figures 4, 5 do not appear in the model proposed in [5].

Next we study relationships between the response types and parameters. We write a response type for each parameter in Figure 6. The presence of phosphorus recycling from the sediment enhances the nonlinear effect in (2.11). Hence the response types II and III arise as $R$ becomes large. This nonlinear effect is reinforced by shortness of $T_{\mathrm{W}}$. Similarly, the hypolimnion enhances the nonlinear effect. By setting $R=5$ and $T_{\mathrm{W}}=80$, for example, the response types are Types I and III in Figures (a) and (c), respectively.

In the last part of this section, we state that a periodic solution of (2.11) can approximate a solution of the system (2.1)-(2.7) by showing response types of (2.1)-(2.7) with the same 

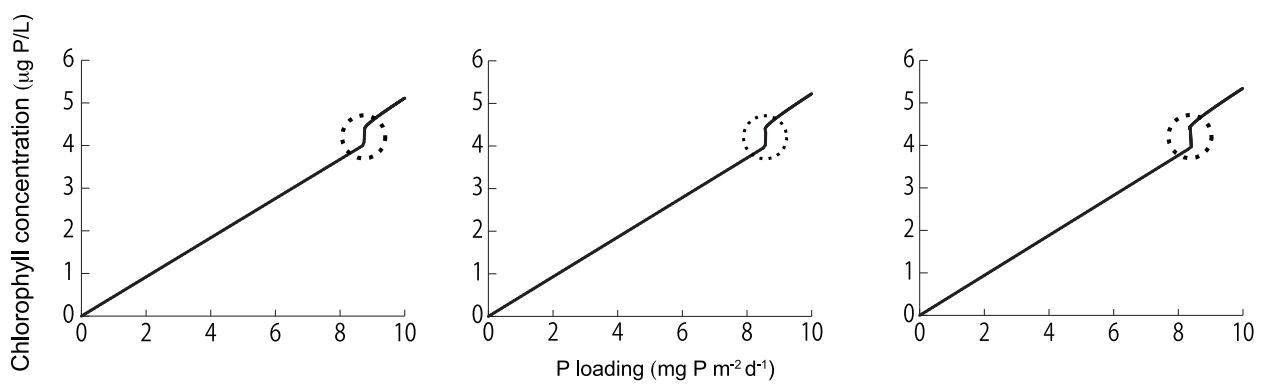

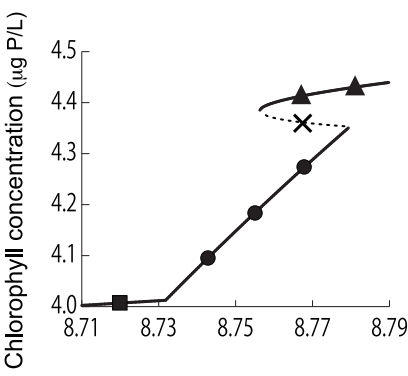

(a) Type IV

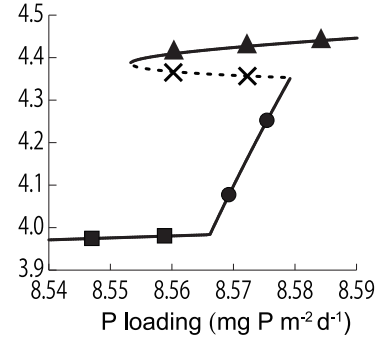

(b) Type V

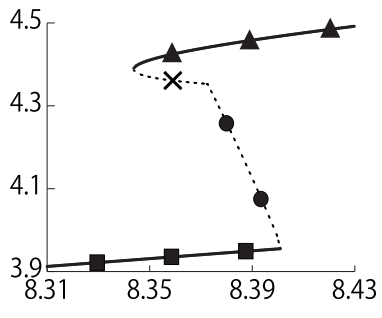

(c) Type VI

Figure 4: Response types IV-VI of (2.11). The horizontal and vertical axes represent the bifurcation parameter $l$ and the fixed points of (3.2). The lines with cross marks correspond to $\bar{X}_{1}^{\text {low }}(l)$. Other marks and two kinds of lines have the the same meanings as in Figure 3 . The parameters of Set II in Table 1 are used. The bottom three figures correspond to the dashed circles in the top ones, respectively. (a) $T_{\mathrm{s}}=95$; (b) $T_{\mathrm{s}}=100$; (c) $T_{\mathrm{s}}=105$.

parameters as in Figure 3 via numerical simulations. For $T_{\mathrm{s}}=244,251,265[\mathrm{~d}]$ and $l \in[0,1]$, we have $X_{k}\left(T_{\mathrm{w}}\right)(k=1, \ldots, 21)$ with a random initial value $\left(P_{0}, X_{0}\right)$. The mesh size of $l$ is 0.01 . We carried out numerical simulations four times for each parameter with different initial values. Calculating an average of $X_{k}\left(T_{\mathrm{w}}\right)$ over $k=12, \ldots, 21$ for each simulation result, we plot all four averages in Figure 7 by putting square marks. In the case of $T_{\mathrm{s}}=244$, four averages have almost the same value for each $l$, which means that the response type of the lake is reversible. In the case of $T_{\mathrm{s}}=251$, large and small averages emerge for each $l \in(0.16,0.42)$, which implies a hysteresis structure occurs and the response type is hysteresis. Finally, in the case of $T_{\mathrm{s}}=$ 265, the response type is irreversible. Therefore we conclude that the increase of the period of summer strongly influences the susceptibility of lakes to regime shits.

\section{Conclusion}

In this article we propose a model for phytoplankton and phosphorus dynamics with 1dimensional vertical structure of lake ecosystem and seasonal periodic cycle of water mixing 

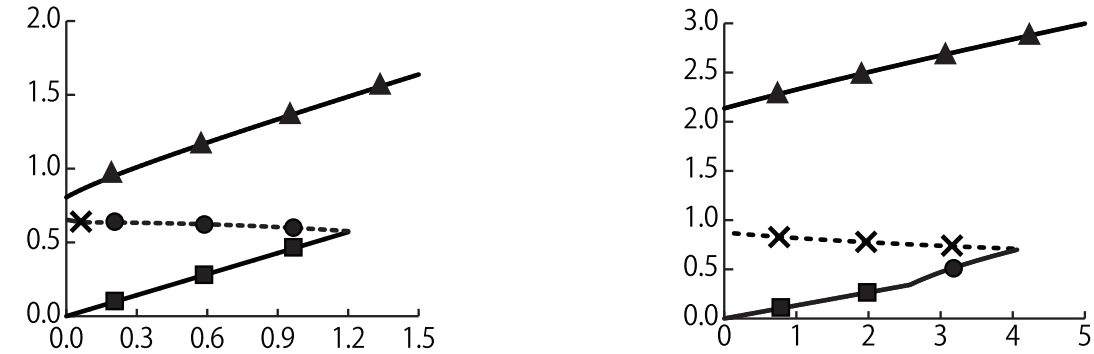

$\mathrm{P}$ loading $\left(\mathrm{mg} \mathrm{P} \mathrm{m}^{-2} \mathrm{~d}^{-1}\right)$

(a) Type VII

(b) Type VIII

Figure 5: Response types VII, VIII of (2.11). The horizontal and vertical axes represents the bifurcation parameter $l$ and the fixed points of (3.2). All marks and two kinds of lines have the the same meanings as in Figures 3, 4. (a) The parameters defined in Set I are used except for $R, T_{\mathrm{s}}, Z_{\mathrm{h}}$. We set $R=9.4, T_{\mathrm{s}}=152, Z_{\mathrm{h}}=297$; (b) $\alpha=1.0, \gamma=1.0, \theta=3.0, \kappa=0.68, R=4000, T_{\mathrm{s}}=$ $118, s_{\mathrm{e}}=s_{\mathrm{h}}=0.27, s_{\mathrm{b}}=7.9, Z_{\mathrm{e}}=275, Z_{\mathrm{h}}=30, Z_{\mathrm{b}}=0.1, \beta_{\mathrm{e}}=\beta_{\mathrm{h}}=\beta_{\mathrm{b}}=\beta=0.0081$.

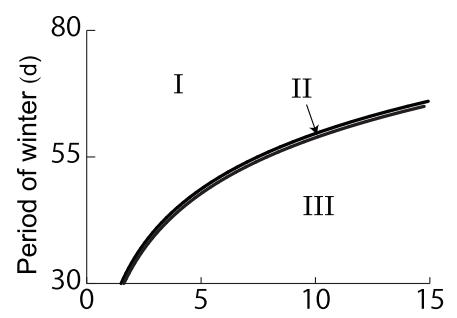

(a)

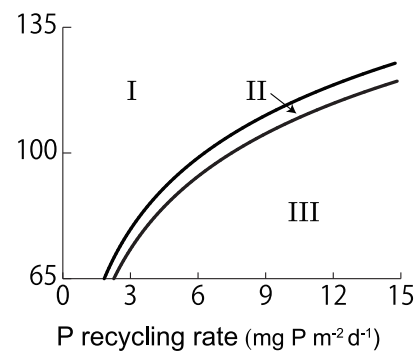

(b)

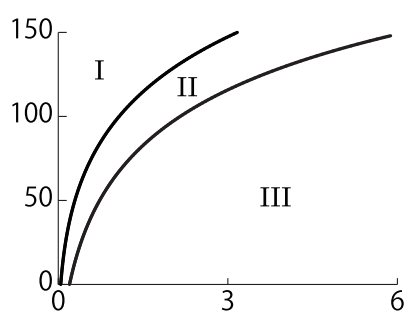

(c)

Figure 6: Relationships between the response types I-III defined in Figure 3 and $\left(R, T_{\mathrm{W}}\right)$. The parameters given by Set I in Table 1 are used except for $R, Z_{\mathrm{h}}, T_{\mathrm{w}}$. (a) $Z_{\mathrm{h}}=30$ [m]; (b) $Z_{\mathrm{h}}=70$ $[\mathrm{m}] ;$ (c) $Z_{\mathrm{h}}=150[\mathrm{~m}]$.

to understand the impact of changes in seasonality in various types of lakes on the occurrence of multiple attractors and hysteresis structure. The horizontal homogeneity is assumed for simplicity. The vertical structure is represented by the epilimnion, the hypolimnion, and the bottom sediment in summer. On the other hand, since convection induces water mixing in the lake, two state variables are assumed to be independent of spatial variables in winter. Through numerical simulations we see that our model can generate multiple periodic solutions. Hence it is shown that there is a hysteresis structure in our model. This fact is almost the same as pointed out in [5].

In order to make our model more mathematically tractable, we derive a reduced sys- 


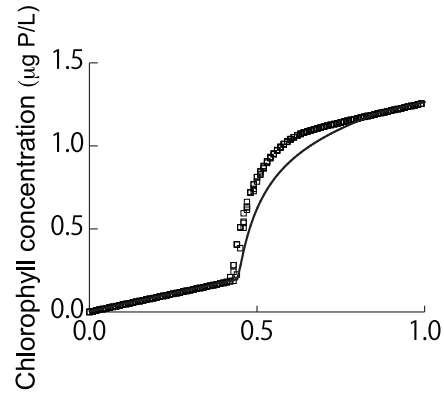

(a) $T_{\mathrm{s}}=244$

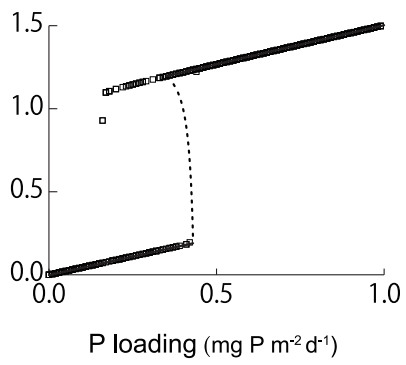

(b) $T_{\mathrm{s}}=251$

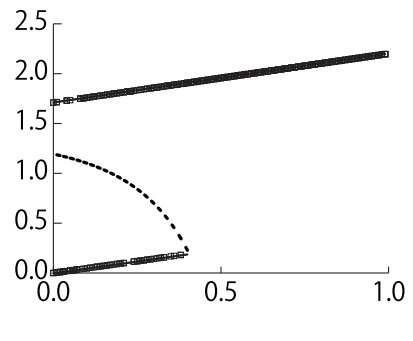

(c) $T_{\mathrm{s}}=265$

Figure 7: Simulation results for (2.1)-(2.7) with the parameters of Set I. We carried out numerical simulations until $t=7665$ (the end of the 21th year) and averaged $X_{k}\left(T_{\mathrm{W}}\right)$ from $k=12$ to $k=21$. We have four results for each $l$ and plot all results. The parameters of Set I are used, and the mesh size of $l$ is 0.01 . The solid and dashed lines are the same as shown in Figure 3 .

tem, which is essentially equivalent to a discrete model (2.11). Under a suitable condition on parameters, this reduced model may approximate the system (2.1)-(2.7). In particular, response types of lakes to eutrophication are almost the same in our original system (see Figure 7). Therefore it suffices to analyze the discrete model instead of the full system.

In the discrete model, we have the existence and stability of fixed points rigorously. Based on those results, we can categorize response types of lakes to eutrophication into eight types. As seen in Figures 3-5, the number of fixed points is either 1 or 3 except for bifurcation points. Finally, we study the relationships among lake response types, seasonality, and P recycling rate in various types of lakes. Figures 6 (a)-(c) show that the shorter period of winter season, larger P recycling rate and deeper hypolimnion strengthen the hysteresis structure. We conclude that the occurrence likelihood of regime shift becomes higher by these factors.

\section{Acknowledgements}

T.M. is supported by Ministry of Science and Technology, Taiwan (MOST 103-2621-M002 -015) and partly by Academia Sinica through a thematic project grant titled "Ocean Acidification: Comparative biogeochemistry in shallow-water tropical coral reef ecosystems in a naturally acidic marine environment".

\section{References}

[1] A. D. Barnosky, E. A. Hadly, J. Bascompte, E. L. Berlow, J. H. Brown, M. Fortelius, W. M. Getz, J. Harte, A. Hastings, P. A. Marquet, and others, Approaching a state shift in Earth's biosphere, Nature, 486 (2012), 52-58.

[2] S. R. Carpenter, D. Ludwig, and W. A. Brock, Management of eutrophication for lakes subject to potentially irreversible change, Ecological applications, 9 (1999), 751-771. 
[3] F. S. Chapin III, C. Folke, and G. P. Kofinas, A framework for understanding change, Principles of ecosystem stewardship, Springer, New York, (2009), 3-28.

[4] C. Folke, S. Carpenter, B. Walker, M. Scheffer, T. Elmqvist, L. Gunderson, and C. S. Holling, Regime shifts, resilience, and biodiversity in ecosystem management, Annual Review of Ecology, Evolution, and Systematics, (2004), 557-581.

[5] M. Genkai-Kato, and S. R. Carpenter, Eutrophication due to phosphorus recycling in relation to lake morphometry, temperature, and macrophytes, Ecology, 86 (2005), 210-219.

[6] M. Scheffer and S. R. Carpenter, Catastrophic regime shifts in ecosystems: linking theory to observation, Trends in ecology \& evolution, 18 (2003), 648-656.

[7] M. Scheffer, S. H. Hosper, M. L. Meijer, B. Moss, and E. Jeppesen, Alternative equilibria in shallow lakes, Trends in ecology \& evolution, 8 (1993), 275-279.

[8] M. Søndergaard, J. P. Jensen, and E. Jeppesen, Role of sediment and internal loading of phosphorus in shallow lakes, Hydrobiologia, 506 (2003), 135-145.

School of Interdisciplinary Mathematical Sciences, Meiji University, 4-21-1, Nakano, Nakano-ku, Tokyo, Japan.

E-mail: ikeda@meiji.ac.jp

Institute of Oceanography, National Taiwan University / Research Center for Environmental Changes, Academia Sinica, No.1 Sec.4 Roosevelt Road, Taipei, Taiwan.

E-mail: tksmiki@ntu.edu.tw 